“ (C) 2016 IEEE. Personal use of this material is permitted. Permission from IEEE must be obtained for all other uses, in any current or future media, including

reprinting/republishing this material for advertising or promotional purposes, creating new collective works, for resale or redistribution to servers or lists, or reuse of any copyrighted component of this work in other works." 


\title{
Model Predictive Control for Single-Phase NPC Converters Based on Optimal Switching Sequences
}

\author{
Sergio Vazquez, Senior Member, IEEE, Ricardo P. Aguilera, Member, IEEE, Pablo Acuna, Member, IEEE, \\ Josep Pou, Senior Member, IEEE, Jose I. Leon, Senior Member, IEEE, Leopoldo G. Franquelo, Fellow, \\ IEEE and Vassilios G. Agelidis, Fellow, IEEE
}

\begin{abstract}
In this paper, a model predictive control (MPC) based on optimal switching sequences (OSSs) for a singlephase grid-connected full-bridge neutral-point-clampled (NPC) power converter is presented. The predictive control algorithm is formulated in terms of OSSs, which was originally proposed to govern three-phase power converters. In this work, the OSS concept is extended to control singlephase power converters. The proposed MPC algorithm belongs to the continuous control set MPC (CCS-MPC) and is able to provide fixed switching frequency while handling system constraints. The proposed algorithm has been experimentally tested in an NPC power converter prototype. Experimental results show the desired fixed switching behavior in the steady state condition and the intrinsic fast dynamic provided by MPC during transients. Furthermore, the test outcomes demonstrate the robustness of the proposed controller under large system parameter deviations.
\end{abstract}

\section{INTRODUCTION}

$\mathbf{P}$ OWER converters play a key role in applications such as integration of renewable energies, energy storage systems, motor drives, etc [1]-[3]. In particular, single-phase power converters have drawn much attention for low-power photovoltaic (PV) integration and railway traction systems [4][6]. For this reason, controller design for single-phase grid connected power converters has an increasing practical value.

The conventional solution to control the input current of power converters is based on linear proportional-integral (PI) regulators working in a synchronous reference frame or proportional plus resonant controllers [7]-[9]. In general, these solutions offer good performance. However, it has been shown that nonlinear control strategies can improve transient

This work was supported by the Ministerio Español de Economia y Competitividad under project ENE2013-45948-R and by the Consejeria de Innovacion Ciencia y Empresa (Junta de Andalucia) under the project P11-TIC-7070.

S. Vazquez, J. I Leon and L. G. Franquelo are with the Electronic Engineering Department, Universidad de Sevilla (Spain), (e-mail: sergi@us.es).

R. P. Aguilera is with the School of Electrical, Mechanical and Mechatronic Systems, University of Technology Sydney, Sydney, NSW 2007 , Australia (e-mail: raguilera@ieee.org).

P. Acuna and V. G. Agelidis are with the University of New South Wales, Sydney, NSW 2052, Australia (e-mail: pabloacunarios@gmail.com; vassilios.agelidis@unsw.edu.au).

J. Pou is with Nanyang Technological University, Singapore 639798 (e-mail: j.pou@ntu.edu.sg). and steady state behavior compared with these conventional approaches [10], [11]. One promising alternative to govern power converters is model predictive control (MPC) [12], [13]. In essence, to implement an MPC strategy, it is firstly required to select a cost function to define a desired control objective. Then, a model of the system is used to forecast its future behavior. Finally, the optimal control action to be applied to the system is the one that minimizes the cost function. This process is repeated at each sampling instant using a receding horizon policy [14]. MPC for power converters can be divided in two major groups depending on the nature of the chosen control input [15]: Finite control set MPC (FCS-MPC) [16] and continuous control set MPC (CCS-MPC) [17].

FCS-MPC takes advantage of the discrete nature of the power converter. In this way, it only calculates the cost function value for the available output voltage vectors of the power converter. For instance, in the case of a conventional single-phase $\mathrm{H}$-bridge inverter, only four voltage vectors are evaluated. The main advantadge of this approach is that the cost function can manage a multi-objective optimization problem and handle system constraints with ease. The main disadvantage of this strategy for controlling grid-connected converters is that it does not provide a constant switching frequency. As a consequence, it is more difficult to design the grid filter. Some alternatives have been presented to solve this problem [18]-[21]. However, this is still an open issue for FCS-MPC.

On the other hand, CCS-MPC considers the control region of the power converter as a continuous space. Therefore, it solves the optimization problem and provides a control action that should be generated by means of a modulator. The main advantage is that it provides a fixed switching frequency. Therefore, this MPC class fits particularly well to control grid-connected power converters. The main problem is that handling system constraints usually requires to solve an optimization quadratic problem. This is highly computational demanding and very difficult to solve online using a conventional digital hardware platform. For this reason, these techniques are usually limited to solve problems without including system constraints [22]-[24].

This paper presents an MPC algorithm, which belongs to the CCS-MPC family, to govern single-phase grid-connected fullbridge neutral-point-clamped (NPC) power converters. The 


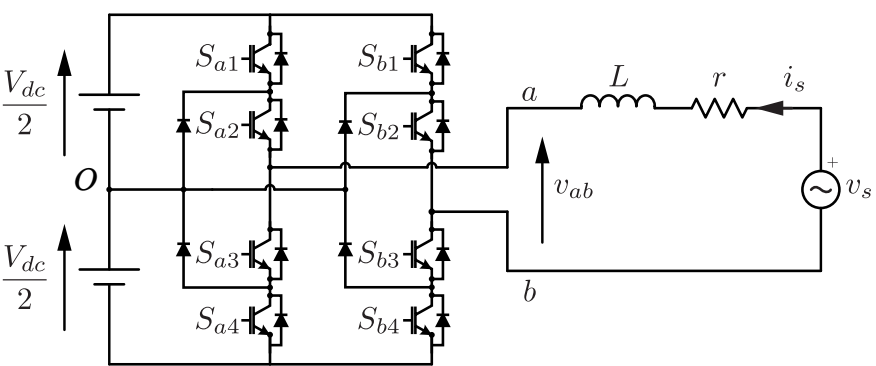

Fig. 1. Circuit diagram of a grid-connected single-phase NPC converter.

proposed method is based on using the optimal switching sequence (OSS) concept to calculate the control action [25]. The main advantage of this approach is that it can handle system constraints while keeping a limited computational burden, hence enabling the implementation of the proposed control algorithm in a standard digital hardware platform. To be more specific, the proposed algorithm evaluates the cost function for each of the available switching sequences for the power converter. Then, like in an FCS-MPC strategy, the one that minimizes the cost function becomes the OSS to be applied in the power converter.

In essence, OSS defines a switching sequence (switching states and corresponding duty cycles) to be applied in the power converter during the next sampling period. The switching sequence in three-phase systems is conventionally calculated implementing a space vector based modulation method. However, in single-phase converters, the usual way to obtain the power switch gate signals is to use a conventional sinusoidal PWM. Since PWM does not relay on the vectorial representation of switching states, a straightforward implementation of the OSS technique by taking into account PWM is not possible. Therefore, a suitable vectorial representation of switching states for single-phase power converters has to be considered first in order to design an OSS-based control algorithm.

In this paper, the basic ideas for developing an OSS-based MPC (OSS-MPC) algorithm for single-phase power converters, focused on the current control tracking, are presented. The proposed OSS-MPC is able to track a reference current, which regulates the transference of power between dc-sources connected to the dc-link and the grid. The proposed algorithm can easily be extended to other configurations but, for the sake of clarity, only the basic concepts are presented using the aforementioned circuit.

The remainder of the paper is organized as follows. Section II shows the model of the grid-connected full-bridge NPC power converter. Section III presents the design of the proposed OSS-MPC strategy. Section IV discusses the selection of a suitable set of switching sequences for the singlephase NPC converter. Section V shows experimental results to validate the proposed control algorithm and finally the main conclusions are summarized in Section VI.

\section{MOdel OF THE SyStem}

The circuit diagram of a single-phase grid-connected fullbridge NPC power converter is depicted in Fig. 1. Here, $S_{x i}$
TABLE I

SYStem VARIABLES AND PARAMETERS

\begin{tabular}{cc}
\hline \hline Variable & Description \\
\hline$v_{s}$ & Grid voltage \\
$i_{s}$ & Grid current \\
$v_{a b}$ & Inverter output voltage \\
$r$ & Output filter resistor \\
$L$ & Output filter inductor \\
$V_{d c}$ & DC-Link voltage \\
\hline \hline
\end{tabular}

TABLE II

Voltage Vectors For the SingLe-PhASE NPC CONVERTER

\begin{tabular}{ccc}
\hline \hline Vector $j$ & $\left(S_{a}, S_{b}\right)$ & $v_{a b j}$ \\
\hline 0 & $(-1,-1)$ & 0 \\
1 & $(0,-1)$ & $\frac{V_{d c}}{2}$ \\
2 & $(1,-1)$ & $V_{d c}$ \\
3 & $(-1,0)$ & $\frac{-V_{d c}}{2}$ \\
4 & $(0,0)$ & 0 \\
5 & $(1,0)$ & $\frac{V_{d c}}{2}$ \\
6 & $(-1,1)$ & $-V_{d c}$ \\
7 & $(0,1)$ & $\frac{-V_{d c}}{2}$ \\
8 & $(1,1)$ & 0 \\
\hline \hline
\end{tabular}

for all $i \in\{1, \ldots, 4\}$ and $x \in a, b$ denotes the, so-called, switching signal to trigger each power switch. Therefore, each of them can take only two values, i.e., $S_{x i} \in\{0,1\}$. Moreover, the pairs of switching signals $S_{x 1}, S_{x 3}$ and $S_{x 2}, S_{x 4}$ work in a complementary way. The variables of the system are shown in Table I. To introduce the concept of OSS, it is assumed that the dc-link voltages are supplied by using external dc sources.

Under this condition, the continuous-time dynamic of the grid current can be described by:

$$
v_{s}=L \frac{d i_{s}}{d t}+r i_{s}+v_{a b},
$$

where the voltage between the output terminals of the power converter is:

$$
v_{a b}=\frac{V_{d c}}{2}\left(S_{a 1}-S_{b 1}+S_{a 2}-S_{b 2}\right) .
$$

Due to the discrete nature of the NPC converter, the output voltage $v_{a b}$ belongs to the following finite control set:

$$
v_{a b} \in \mathbb{V}_{a b}=\left\{-V_{d c},-\frac{V_{d c}}{2}, 0, \frac{V_{d c}}{2}, V_{d c}\right\} .
$$


These voltage levels can be generated by redefining (2) as:

$$
v_{a b}=\frac{V_{d c}}{2}\left(S_{a}-S_{b}\right),
$$

with $S_{a}$ and $S_{b}$ representing the switching functions for the legs $a$ and $b$, respectively. Thus, each element of the pair $\left(S_{a}, S_{b}\right)$, which can individually take only three values, defines the available finite control sets for the single-phase full-bridge NPC converter as:

$$
\begin{aligned}
& S_{a} \in\{-1,0,1\}, \\
& S_{b} \in\{-1,0,1\} .
\end{aligned}
$$

Table II summarizes the nine output voltages that can be generated using the corresponding voltage vectors $v_{a b j}$ with $j \in\{0 \cdots 8\}$.

\section{Optimal Switching Sequence Strategy}

To design the proposed OSS-MPC strategy for the singlephase full-bridge NPC converter, (1) can be rewritten as:

$$
\frac{d i_{s}}{d t}=\frac{1}{L}\left(v_{s}-r i_{s}-v_{a b}\right),
$$

which represents the variation of the grid current in a switching interval for a given converter output voltage $v_{a b}$. Therefore, for each $v_{a b j} \in \mathbb{V}_{a b}$, it is possible to define a function that describes the variation of the grid current, $i_{s}$, as:

$$
f_{i_{s}}\left(v_{a b}\right)=\left.\frac{d i_{s}}{d t}\right|_{v_{a b}=v_{a b j}} \quad \forall j \in\{0 \cdots 8\} .
$$

Consider a switching sequence

$$
S e q_{m}=\left\{v_{a b j, 1}, v_{a b k, 2}, v_{a b l, 3}\right\}
$$

with $j, k, l \in\{0 \cdots 8\}$ and $m \in \mathbb{N}$. The output voltages $v_{a b j, 1}, v_{a b k, 2}$, and $v_{a b l, 3}$ are applied sequentially during the time intervals $t_{1}, t_{2}$ and $t_{3}$, respectively, where

$$
t_{1}+t_{2}+t_{3}=T_{s},
$$

in which $T_{s}$ stands for the sampling period. The time set, $T_{S e q_{m}}$, corresponding with $S e q_{m}$ can be defined as $T_{S e q_{m}}=$ $\left\{t_{1}, t_{2}, t_{3}\right\}$. Then, the sequence $S e q_{m}$ has associated the set

$$
\left.F_{i_{s}}\right|_{S e q_{m}}=\left\{f_{i_{s} 1}, f_{i_{s} 2}, f_{i_{s} 3}\right\},
$$

calculated from applying $S e q_{m}$ to (8). With these values, and assuming that the sampling frequency is high enough, then the measured values of $i_{s, k}$ and $v_{s, k}$ at each sampling instant $k$ are assumed to remain constant during the interval $T_{s}$. Therefore, from (7) and (8), a prediction for the grid current at the end of the sampling period can be computed as:

$$
\left.\hat{i}_{s, k+1}\right|_{S e q_{m}}=i_{s, k}+\sum_{n=1}^{n=3} f_{i_{s} n} t_{n}
$$

where $\hat{i}_{s, k+1}$ and $i_{s, k}$ stand for the predicted and actual current at instants $k+1$ and $k$, respectively. An illustrative example of the proposed concept showing the evolution of the predicted current trajectories from the beginning to the end of a sampling period is shown in Fig. 2.

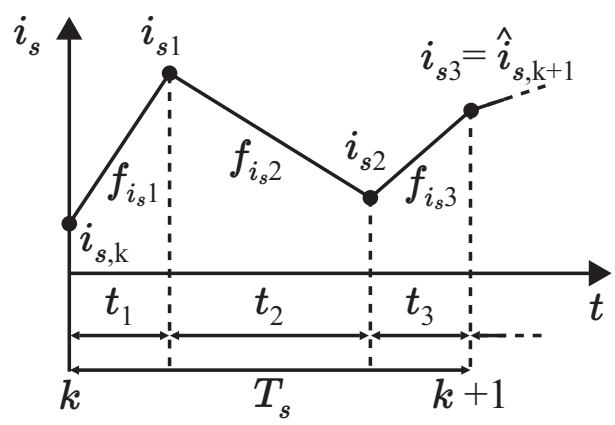

Fig. 2. Predicted current trajectories $\left(i_{s 1}, i_{s 2}\right.$ and $\left.i_{s 3}\right)$ for a switching sequence of three vectors.

\section{A. Cost Function Formulation}

The proposed OSS-MPC can be designed using (12). For this purpose, the current error at the instant $k+1$ is defined as

$$
e_{i, k+1}=i_{s, k+1}^{*}-\hat{i}_{s, k+1},
$$

$i_{s, k+1}^{*}$ being the current reference. Substituting (12) in (13) yields

$$
e_{i, k+1}=e_{i 0}-\sum_{n=1}^{n=3} f_{i_{s} n} t_{n}
$$

where $e_{i 0}=i_{s, k+1}^{*}-i_{s, k}$. Taking into account (10), the expression for the current error of each sequence $S e q_{m}$ becomes

$$
e_{i, k+1}=e_{i 0}-\left(\left(f_{i_{s} 1}-f_{i_{s} 3}\right) t_{1}+\left(f_{i_{s} 2}-f_{i_{s} 3}\right) t_{2}+f_{i_{s} 3} T_{s}\right) .
$$

A simple cost function considering the quadratic current error is adopted as

$$
J=e_{i, k+1}^{2} .
$$

Other cost functions can be considered. For instance, if only one dc voltage source is employed and the converter handles the dc-link capacitor balancing problem, then the cost function

$$
J=e_{i, k+1}^{2}+\lambda e_{\Delta v_{C}, k+1}^{2}
$$

could be used, where $e_{\Delta v_{C}, k+1}$ stands for the dc-link capacitor voltages difference error at instant $k+1$ and $\lambda$ is a weighting factor. However, to introduce the concept of the proposed control strategy, in this work $J$ is chosen as defined in (16) to be as simple as possible for the sake of clarity. This cost function allows one to track a desired current reference while the dc-link capacitor voltage balance problem can be solved by using a voltage vector redundancy approach [11], [26].

The time values $T_{S e q_{m}}=\left\{t_{1}, t_{2}, t_{3}\right\}$ that minimize the cost function $J$ using the voltage sequence $S e q_{m}=$ $\left\{v_{a b j, 1}, v_{a b k, 2}, v_{a b l, 3}\right\}$ can be calculated solving the system

$$
\begin{aligned}
& \left.\frac{\partial J}{\partial t_{1}}\right|_{S e q_{m}}=0, \\
& \left.\frac{\partial J}{\partial t_{2}}\right|_{S e q_{m}}=0 .
\end{aligned}
$$

Once $T_{S e q_{m}}$ is calculated, it is possible to determine the cost value, $J_{m}$, when a particular sequence $S e q_{m}$ is evaluated,

$$
J_{m}=\left.J\right|_{S e q_{m}} .
$$


As each sequence $S e q_{m}$ provides a different cost value, $J_{m}$, then an FCS-MPC fashion approach can be used to obtain the optimal global solution. In this way, the OSS, Seqopt, is thus defined as

$$
S e q_{o p t}=\left\{v_{a b j, 1}, v_{a b k, 2}, v_{a b l, 3}\right\}_{o p t},
$$

which is the one that minimizes $J$, i.e.,

$$
S e q_{o p t}=\arg \min _{S e q_{m}} J
$$

with the associated optimal time set, $T_{o p t}$, which has the form

$$
T_{\text {opt }}=\left\{t_{1}, t_{2}, t_{3}\right\}_{\text {opt }} .
$$

\section{B. Handling System Constraints}

Assuming that the maximum current the power switches in the converter can stand is $I_{\max }$, then, the cost function (16) is modified to account for this system constraint as follows:

$$
J^{\prime}=J+J_{I_{\max }}
$$

where $J_{I_{\max }}$ is defined as

$$
J_{I_{\max }}=\left\{\begin{array}{lll}
0 & \text { if } & \hat{i}_{s, k+1} \leq I_{\max } \\
\infty & \text { if } & \hat{i}_{s, k+1}>I_{\max } .
\end{array}\right.
$$

It should be noticed that

$$
\begin{aligned}
& \left.\frac{\partial J^{\prime}}{\partial t_{1}}\right|_{S e q_{m}}=\left.\frac{\partial J}{\partial t_{1}}\right|_{S e q_{m}}, \\
& \left.\frac{\partial J^{\prime}}{\partial t_{2}}\right|_{S e q_{m}}=\left.\frac{\partial J}{\partial t_{2}}\right|_{S e q_{m}} .
\end{aligned}
$$

Therefore, the time set $T_{S e q_{m}}$ calculated from (18) and (19) is the same that minimizes $J^{\prime}$. Once $T_{S e q_{m}}$ is calculated, the value of $J^{\prime}$ can be evaluated. Thus, for a given sequence $S e q_{m}$ the cost function value $J_{m}^{\prime}$ is

$$
J_{m}^{\prime}=\left.J^{\prime}\right|_{S e q_{m}},
$$

and the optimization problem to be solved is transformed to

$$
S e q_{o p t}=\arg \min _{S e q_{m}} J^{\prime},
$$

with the associated optimal time set, $T_{o p t}$.

\section{SWITCHING SEQUENCE DEFINITION}

The problem solved in (28) requires a suitable set of switching sequences to be evaluated in order to find the optimal one. Defining this set can be addressed considering the discrete nature of the output voltage vectors generated by the NPC single-phase converter. An approach similar to the two-dimensional modulation technique can be used to describe the control region [27]-[29].

The control region associated to the single-phase NPC converter is represented in Fig. 3. In this plot, the nine available output voltage vectors are defined by the pair $\left(S_{a}, S_{b}\right)$. Several voltage sequences can be defined in the control region depicted in Fig. 3. However, a simple approach will be described in this work for the sake of clarity. Further analysis of this issue will be addressed in future work.

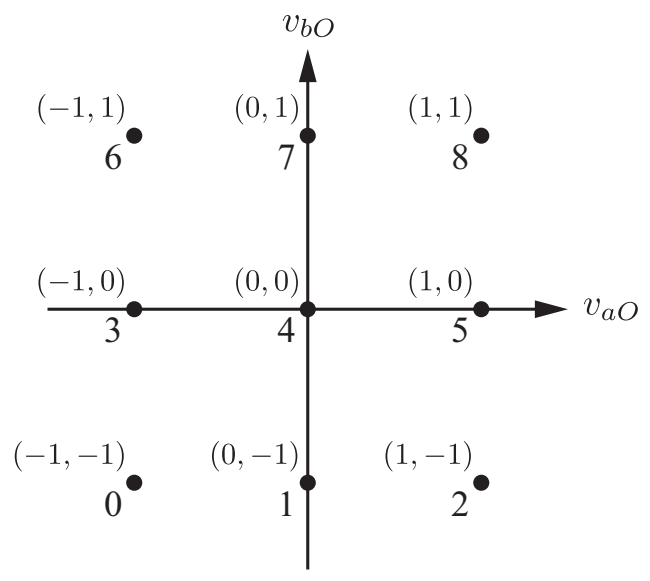

Fig. 3. Control region showing the discrete output voltage vectors generated by the single-phase full-bridge NPC converter.

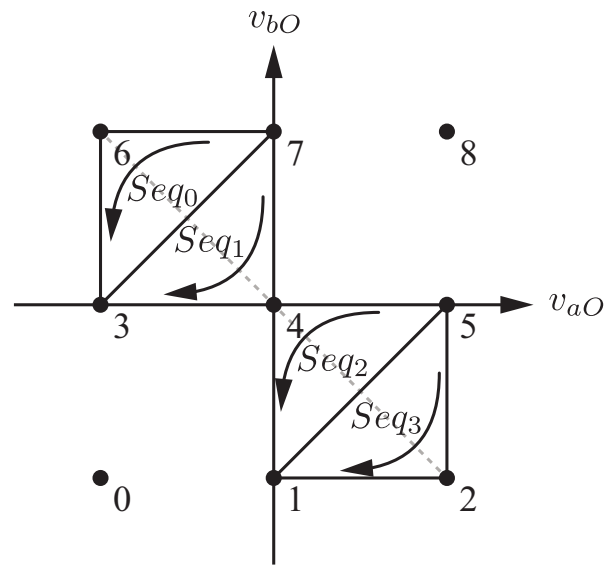

Fig. 4. Proposed switching sequences generated by combining three output voltage vectors.

\section{A. A Simple Switching Sequence Definition}

In order to define the switching sequences, it is important to analyze the problem to be solved. The first step is to find the application times, $T_{S e q_{m}}$, for a given switching sequence, $S e q_{m}$ as per (9). Recalling (25) and (26), and considering (15), for a given sequence the system is reduced to

$$
e_{i 0}-\left(f_{i_{s} 1}-f_{i_{s} 3}\right) t_{1}-\left(f_{i_{s} 2}-f_{i_{s} 3}\right) t_{2}-f_{i_{s} 3} T_{s}=0 .
$$

From (29), it can be observed that the solution for the system is not unique. Therefore, an additional constraint can be included to find a straightforward solution to determine the switching sequences.

If $S e q_{o p t}$ and $T_{o p t}$ were defined, then an equivalent control signal $v_{m}$ could be calculated as

$$
v_{m}=\frac{1}{T_{s}}\left(v_{a b j, 1} t_{1}+v_{a b k, 2} t_{2}+v_{a b l, 3} t_{3}\right),
$$

where $\left\{v_{a b j, 1}, v_{a b k, 2}, v_{a b l, 3}\right\}$ and $\left\{t_{1}, t_{2}, t_{3}\right\}$ are taken from the sets $S e q_{o p t}$ and $T_{o p t}$, respectively.

Taking into account that a degree of freedom exists, the control region is divided, as seen in Fig. 4, in order to achieve a similar behavior of the single-phase full-bridge NPC converter as if modulated by unipolar PWM scheme. In that case, the equivalent control signal, $v_{m}$, should move 
TABLE III

SWITCHING SEQUENCES

\begin{tabular}{cc}
\hline \hline Switching sequence & Voltage vector sequence \\
\hline$S e q_{0}$ & $\{7,6,3\}$ \\
$S e q_{1}$ & $\{7,4,3\}$ \\
$S e q_{2}$ & $\{5,4,1\}$ \\
$S e q_{3}$ & $\{5,2,1\}$ \\
\hline \hline
\end{tabular}

TABLE IV

SWITCHING DUty CyCLES For EACH SWITCHING SEQUENCE

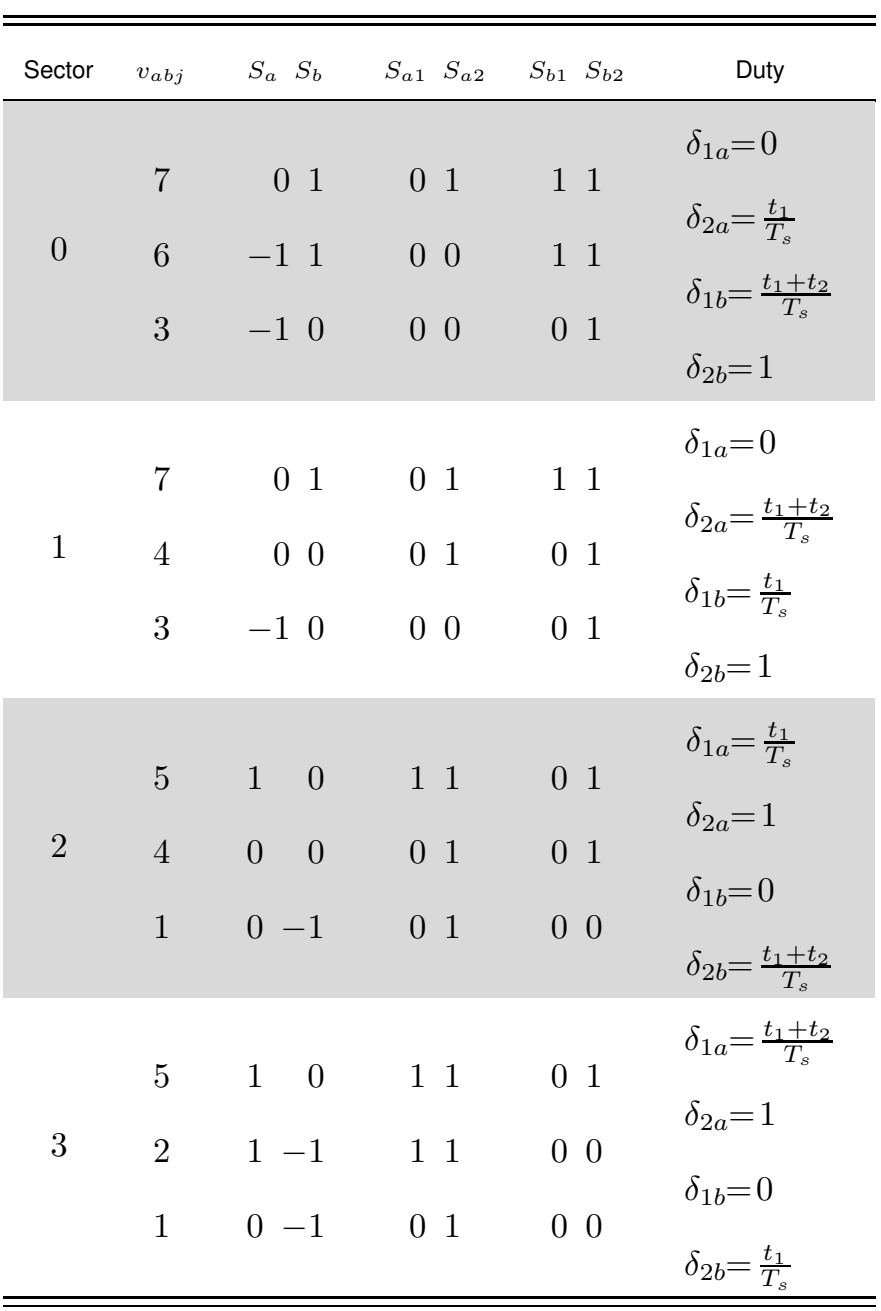

along the discontinuous grey line in Fig. 4. Therefore, only four switching sequences, $S e q_{j}$, with $j \in\{1, \ldots, 4\}$ are needed to synthesize $v_{m}$. The switching sequences for this approach are shown in Table III. These switching sequences and their associated sector are depicted in Fig. 4. In addition, a proper path and direction to go through the selected switching sequences has been chosen, as described by the arrows in Fig. 4.

Under the assumption that $v_{m}$ moves on the dashed grey line in Fig. 4, then the voltage vectors $v_{a b j, 1}$ and $v_{a b l, 3}$ should be applied during the same time duration, i.e., $t_{3}=t_{1}$. In this

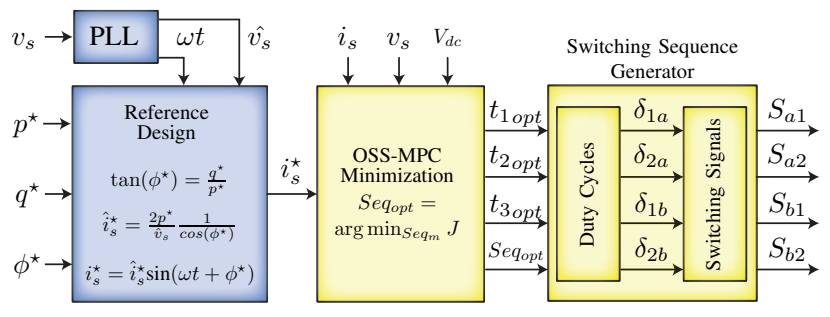

Fig. 5. Block diagram of the proposed OSS-MPC strategy.

way, it is ensured that $v_{m}$ is located over the desired line in the control region. Using this constraint, the system (29) can be solved and the application times for each voltage vector in the switching sequence are

$$
\begin{aligned}
& \left.t_{1}\right|_{S e q_{m}}=\frac{e_{i 0}-f_{i_{s} 2} T_{s}}{\left(f_{i_{s} 1}-2 f_{i_{s} 2}+f_{i_{s} 3}\right)}, \\
& \left.t_{2}\right|_{S e q_{m}}=T_{s}-2 t_{1}, \\
& \left.t_{3}\right|_{S e q_{m}}=t_{1} .
\end{aligned}
$$

Then, the OSS is determined by solving (28) as described in Section III. The implementation of the new switching sequences can be performed as described in Table IV, where $\delta_{1 a}$ and $\delta_{2 a}$ stand for the duty cycles of the power switches $S_{a 1}$ and $S_{a 2}$ in leg $a$ in Fig. 1, respectively, while $\delta_{1 b}$ and $\delta_{2 b}$ represent the duty cycles for the power switches $S_{b 1}$ and $S_{b 2}$ in leg $b$ in Fig. 1, respectively. A block diagram of the proposed OSS-MPC strategy is presented in Fig. 5

\section{EXPERIMENTAL RESULTS}

Experimental results of a single-phase three-level NPC converter governed by the proposed OSS-MPC strategy are presented in this section to validate the effectiveness and performance of the proposed controller. The following results have been obtained with the parameters showed in Table $\mathrm{V}$ by using the experimental setup presented in Fig. 6.

In general, MPC algorithms have a significant computational burden. However, due to the development in processing power of control hardware, predictive control algorithms can be implemented in standard hardware platforms [30]. The proposed OSS-MPC particularly performs the number of operations reflected in Table VI. Although this amount of computations is not small, they can be executed in real time in any standard control hardware platform. For the experiments, the control algorithm was implemented in a DS1106 DSPACE platform, while the switching sequence were generated in a DS5203 FPGA board. The FPGA board provides the interruption signal to the processor at a predetermined sampling frequency $f_{s}=1 / T_{s}$. In response to the interruption signal, the control algorithm generates the switching duty cycles that returns into the FPGA to finally firing the NPC switches according to Table IV. Since $t_{1}$ and $t_{3}$ have been considered to be equal, a symmetrical switching pattern [31] between two consecutive switching sequences is used. The symmetrical switching pattern allows one to implement a faster sampling 
TABLE V

SYSTEM PARAMETERS

\begin{tabular}{cccc}
\hline \hline Variable & Description & Value & p.u. \\
\hline$S_{r}$ & Total rated apparent power & $2.5 \mathrm{kVA}$ & 1 \\
$v_{s}$ & grid-voltage & $230 \mathrm{~V}$ & 1 \\
$V_{d c}$ & dc capacitor voltage & $400 \mathrm{~V}$ & 1 \\
$L$ & Filter inductor & $8 \mathrm{mH}$ & 0.11 \\
$r$ & Filter resistance & $179 \mathrm{~m} \Omega$ & 0.0056 \\
$f_{s}$ & Sampling frequency & $10 \mathrm{kHz}$ & 1 \\
$f_{s w}$ & Switching frequency per leg & $5 \mathrm{kHz}$ & 0.5 \\
\hline \hline
\end{tabular}

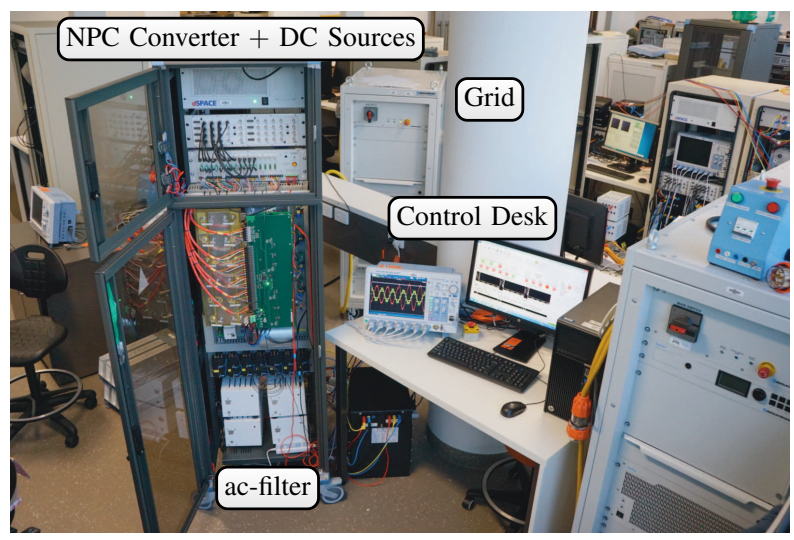

Fig. 6. 2.5 kVA experimental prototype.

TABLE VI

Operations Performed by the OSS-MPC ALGorithm

\begin{tabular}{cc}
\hline \hline Operation type & Quantity \\
\hline Sum & 80 \\
Product & 56 \\
Division & 4 \\
\hline \hline
\end{tabular}

frequency, resulting in more accurate predictions while reducing twice the desired switching frequency per leg $\left(f_{s w}\right)$. Thus, all the results reported in this section were designed to obtain a fixed $f_{s w}$ of $5 \mathrm{kHz}$.

\section{A. Dynamic Performance}

As an initial test, a step change in the amplitude of the current reference $i_{s}^{\star}$ from $10 \mathrm{~A}$ to $15 \mathrm{~A}$ is introduced as depicted in Fig. 7. This figure includes the grid voltage, $v_{s}$, which is affected by the current ripple due to the fact that $v_{s}$ comes from a non-stiff low voltage ac source. Since implementing the proposed control algorithm requires the measured value of $v_{s, k}$, this voltage is obtained through a second order generalized integrator (SOGI) single-phase phase-locked-loop [32]. The output of this block provides a reconstructed version of $v_{s, k}$ which is free of harmonic distortion, and therefore useful to run predictions. The converter output voltage shown (a)

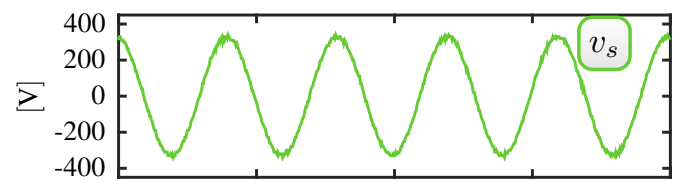

(b)
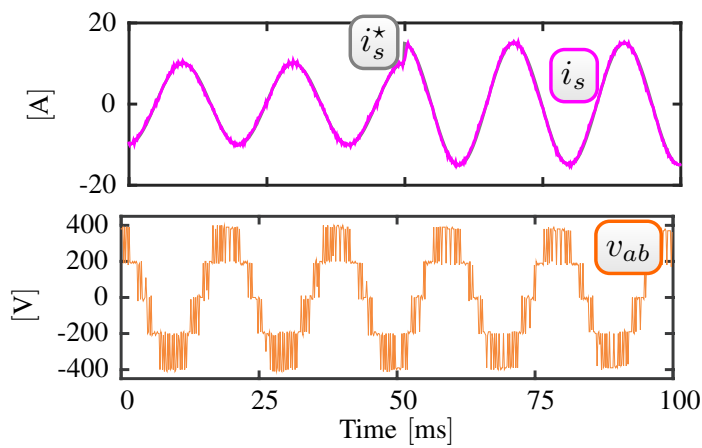

Fig. 7. Step change of $i_{s}$ at $50 \mathrm{~ms}$ from $10 \mathrm{~A}$ to $15 \mathrm{~A}$ under OSS-MPC. (a) Grid voltage, (b) Grid current and (c) Power converter output voltage.

(a)

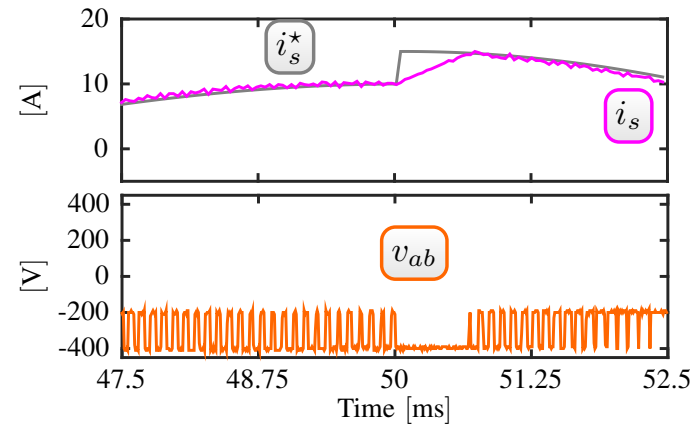

Fig. 8. Zoom of Fig. 7 between $47.5 \mathrm{~ms}$ to $52.5 \mathrm{~ms}$ under OSS-MPC. (a) Grid current and its reference and (b) Converter output voltage.

in Fig. 7(c) presents a three-level waveform as expected, where no undesired spikes in the voltage are observed.

In Fig. 8, a zoom view of the results of the OSS-MPC test is depicted. Fig. 8(a) shows that the grid current effectively tracks its reference when a step change is applied. In this case, the controller behaves correctly by forcing to apply the switching sequence that produces the lowest available output $(-400 \mathrm{~V})$, as shown in Fig. 8(b). Moreover, a response of $1 \mathrm{~ms}$ in the tracking of the current is achieved when a step change of 1.5 p.u. is applied.

For comparison purpose, the same test is performed when the NPC converter is governed by a standard FCS-MPC and a traditional Resonant-Proportional Controller (RPC) adopted from [11] and [9] respectively. The closed-loop performance obtained when using FCS-MPC is depicted in Fig. 9. Here, one can observe that the current also achieves its reference in $1 \mathrm{~ms}$ (see Fig. 9(a)). Therefore, the proposed control strategy is able to provide a fast closed-loop dynamic that matches the one obtained with FCS-MPC. In addition, Fig. 10 and Fig. 11 show the results for the same test when the the converter is governed by RPC. A damping factor $\xi$ equal to 0.707 , and two different close-loop band width (BW) equal to $550 \mathrm{~Hz}$ and $1100 \mathrm{~Hz}$ were selected. It can be noticed that the RPC with larger BW produces a large overshoot (see Fig. 11(a)), which is not observed with the proposed OSS-MPC. To mitigate this, an RPC with lower BW can be implemented. However, as 
(a)

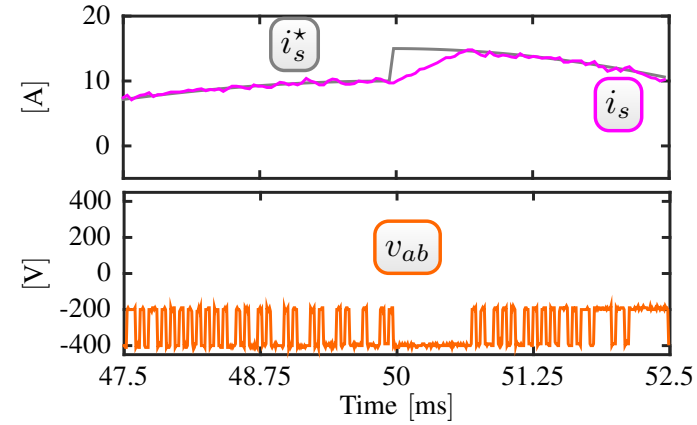

Fig. 9. Standard FCS-MPC. (a) Grid current and its reference and (b) Converter output voltage.

(a)

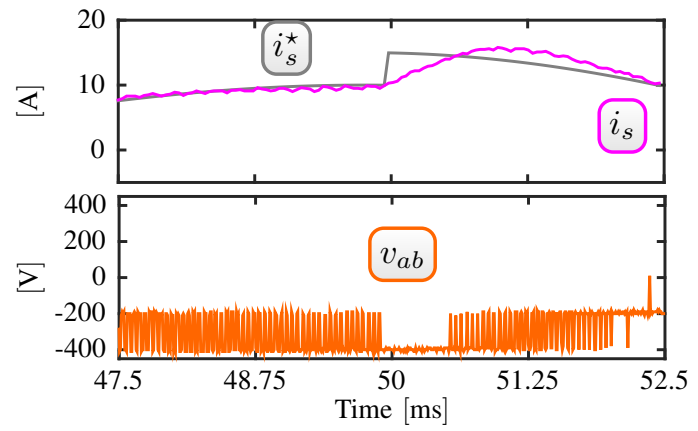

Fig. 10. Resonant Proportional controller with $\mathrm{BW}=550 \mathrm{~Hz}$ and $\zeta$ $=0.707$. (a) Grid current and its reference and (b) Converter output voltage.

(a)

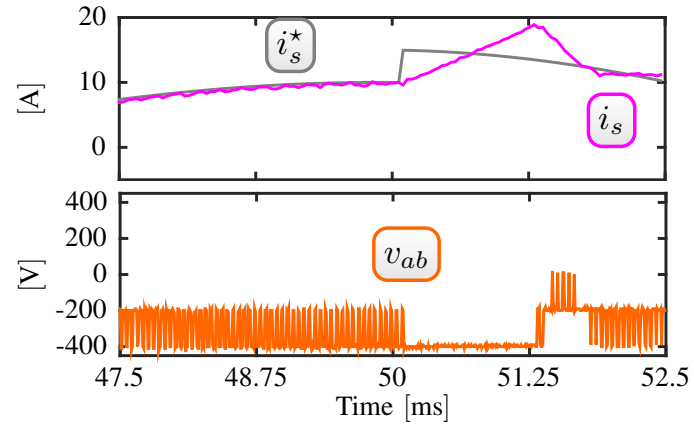

Fig. 11. Resonant Proportional controller with $\mathrm{BW}=1100 \mathrm{~Hz}$ and $\zeta$ $=0.707$. (a) Grid current and its reference and (b) Converter output voltage.

expected, this leads to a low dynamic response as shown in Fig. 10(a). The details about the RPC implementation have been omitted for the sake of brevity, but can be found in [9].

\section{B. Steady State Performance}

In Fig. 12, experimental results of the proposed control strategy in the steady state operation are presented. A nearly sinusoidal grid-current is achieved, while the associated spectrum presents harmonics around $10 \mathrm{kHz}$ which is twice the switching frequency per leg. The harmonic content in the grid current at frequencies below $10 \mathrm{kHz}$ are characterized by a magnitude less than $0.25 \%$ of the fundamental component. Ideally, the third and fifth harmonics should be cancelled. However, the harmonic content of the current depends not only on the current tracking but also on the harmonics presented in the reference signals. Clearly, an optimization of the singlephase PLL may be carried out to reduce the lower harmonics. (a)

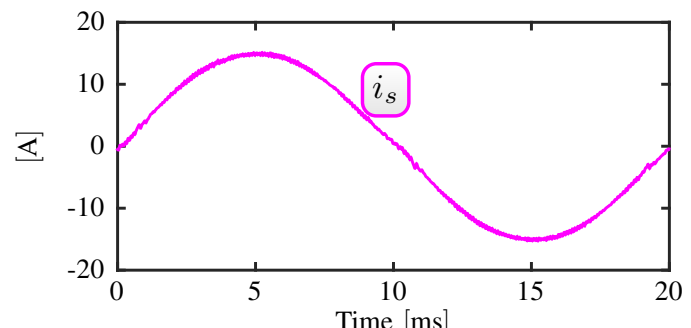

(b)

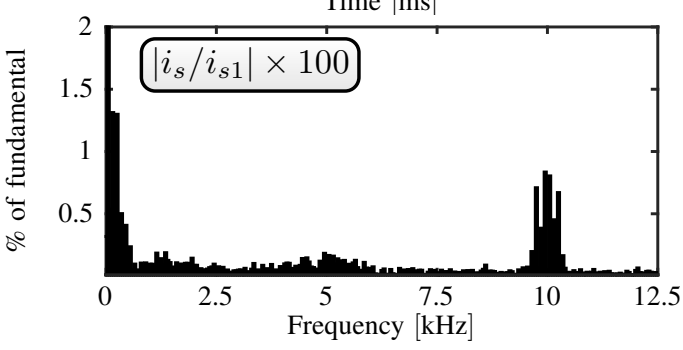

Fig. 12. Steady state analysis. (a) Grid current and (b) Frequency spectrum of the grid current.

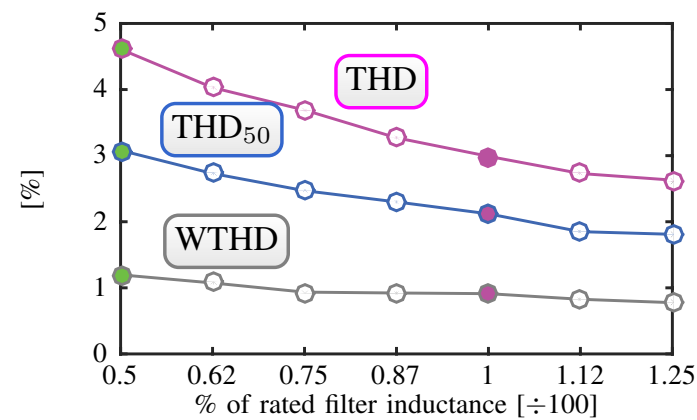

(a)

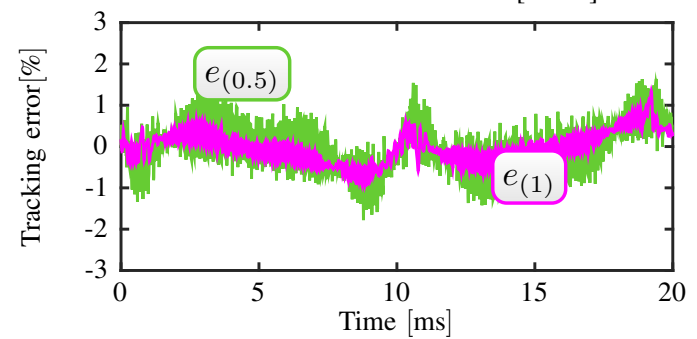

Fig. 13. Steady state distortion of the grid-current under filter inductance value $(L)$ variation. (a) Distortion indices and (b) Instantaneous current tracking error $\left(e_{(L)}=\left(i_{s}-i_{s}^{\star}\right) / i_{s 1} \times 100\right)$ for $L$ equal to $8 \mathrm{mH}$ (1) and $4 \mathrm{mH}(0.5)$.

Nevertheless, the harmonic content is still in concordance with the recommendations of the IEEE Std. 519, [33].

\section{Parameters' Sensitivity}

It follows from (8) and (15) that the proposed OSS-MPC requires information about the output filter to carry out prediction calculations needed to find the OSS. Therefore, it is important to test the controller behavior under parameter uncertainties. As a final test, inductance value variations were performed in order to experimentally obtain the distortion indices related with uncertainty in the output filter value. Fig. 13(a) summarizes the distortion indices obtained when the output filter changes, but the controller is still set to work with the rated value of $L$. Two types of distortion indices are used. The total harmonic distortion (THD) and THD until the $50^{\text {th }}$ harmonic $\left(\mathrm{THD}_{50}\right)$. The results show that the indices 
raise close to $5 \%$ when the actual inductance is $50 \%$ of the rated value. The controller responds to the lack of accurate information by increasing the tracking error as shown in Fig. 13(b). For this reason, the small variation of weighted THD (WTHD) around 1 is useful to show that uncertainties in $L$ affect the tracking error but still the controller behaves as intended by pushing the lowest harmonics near to the switching frequency.

\section{CONCLUSION}

In this work, the optimal switching sequence MPC (OSSMPC) algorithm focused on the current control loop for singlephase full-bridge NPC power converters has been presented. The proposed OSS-MPC belongs to the continuous control set MPC (CCS-MPC) family and makes use of the switching sequence concept in its formulation in order to achieve the optimal control action providing fixed switching frequency. To achieve this, a vectorial representation of switching states for single-phase power converters has been addressed in order to facilitate the design of the proposed OSS-MPC. Experimental results have validated the proposed algorithm including the analysis of transient and steady state performance. Both have shown that the proposed OSS-MPC provides a fast closedloop dynamic performance that matches the one obtained with standard FCS-MPC but with the advantage of obtaining a well-defined and concentrated current spectrum. Robustness of the proposed strategy under system parameter uncertainties has been also tested. Practical results have shown that the proposed controller performs well even under large parameter deviations.

\section{REFERENCES}

[1] J. M. Carrasco, L. G. Franquelo, J. T. Bialasiewicz, E. Galvan, R. C. P. Guisado, M. A. M. Prats, J. I. Leon, and N. MorenoAlfonso, "Power-electronic systems for the grid integration of renewable energy sources: A survey," IEEE Trans. Ind. Electron., vol. 53, DOI 10.1109/TIE.2006.878356, no. 4, pp. 1002-1016, Jun. 2006.

[2] S. Vazquez, S. M. Lukic, E. Galvan, L. G. Franquelo, and J. M. Carrasco, "Energy storage systems for transport and grid applications," IEEE Trans. Ind. Electron., vol. 57, DOI 10.1109/TIE.2010.2076414, no. 12, pp. 3881-3895, Dec. 2010.

[3] M. P. Kazmierkowski, L. G. Franquelo, J. Rodriguez, M. A. Perez, and J. I. Leon, "High-performance motor drives," IEEE Ind. Electron. Mag., vol. 5, DOI 10.1109/MIE.2011.942173, no. 3, pp. 6-26, Sep. 2011.

[4] S. Kouro, J. I. Leon, D. Vinnikov, and L. G. Franquelo, "Gridconnected photovoltaic systems: An overview of recent research and emerging pv converter technology," IEEE Ind. Electron. Mag., vol. 9, DOI 10.1109/MIE.2014.2376976, no. 1, pp. 47-61, Mar. 2015.

[5] E. Romero-Cadaval, G. Spagnuolo, L. G. Franquelo, C. A. Ramos-Paja, T. Suntio, and W. M. Xiao, "Grid-connected photovoltaic generation plants: Components and operation," IEEE Ind. Electron. Mag., vol. 7, DOI 10.1109/MIE.2013.2264540, no. 3, pp. 6-20, Sep. 2013.

[6] A. Steimel, "Electric railway traction in europe," IEEE Ind. Appl. Mag., vol. 2, DOI 10.1109/2943.541241, no. 6, pp. 6-17, Nov. 1996.

[7] C. B. Jacobina, E. C. dos Santos, N. Rocha, and E. L. Lopes Fabricio, "Single-phase to three-phase drive system using two parallel single-phase rectifiers," IEEE Trans. Power Electron., vol. 25, DOI 10.1109/TPEL.2009.2037420, no. 5, pp. 1285-1295, May. 2010.

[8] R. Teodorescu, F. Blaabjerg, M. Liserre, and P. C. Loh, "Proportionalresonant controllers and filters for grid-connected voltage-source converters," Electric Power Applications, IEE Proceedings, vol. 153, no. 5, pp. 750-762, Sep. 2006.

[9] P. Lezana, C. A. Silva, J. Rodriguez, and M. A. Perez, "Zero-steadystate-error input-current controller for regenerative multilevel converters based on single-phase cells," IEEE Trans. Ind. Electron., vol. 54, DOI 10.1109/TIE.2007.891994, no. 2, pp. 733-740, Apr. 2007.
[10] D. Karagiannis, E. Mendes, A. Astolfi, and R. Ortega, "An experimental comparison of several pwm controllers for a singlephase ac-dc converter," IEEE Trans. Control Syst. Technol., vol. 11, DOI 10.1109/TCST.2003.815549, no. 6, pp. 940-947, Nov. 2003.

[11] P. Acuna, L. Moran, M. Rivera, R. Aguilera, R. Burgos, and V. G. Agelidis, "A single-objective predictive control method for a multivariable single-phase three-level npc converter-based active power filter,' IEEE Trans. Ind. Electron., vol. 62, DOI 10.1109/TIE.2015.2393556, no. 7, pp. 4598-4607, Jul. 2015.

[12] S. Kouro, P. Cortes, R. Vargas, U. Ammann, and J. Rodriguez, "Model predictive control: A simple and powerful method to control power converters," IEEE Trans. Ind. Electron., vol. 56, DOI 10.1109/TIE.2008.2008349, no. 6, pp. 1826-1838, Jun. 2009.

[13] S. Vazquez, J. I. Leon, L. G. Franquelo, J. Rodriguez, H. A. Young, A. Marquez, and P. Zanchetta, "Model predictive control: A review of its applications in power electronics," IEEE Ind. Electron. Mag., vol. 8, DOI 10.1109/MIE.2013.2290138, no. 1, pp. 16-31, Mar. 2014.

[14] P. Cortes, M. P. Kazmierkowski, R. M. Kennel, D. E. Quevedo, and J. Rodriguez, "Predictive control in power electronics and drives," IEEE Trans. Ind. Electron., vol. 55, DOI 10.1109/TIE.2008.2007480, no. 12, pp. 4312-4324, Dec. 2008.

[15] D. E. Quevedo, R. P. Aguilera, and T. Geyer, Advanced and Intelligent Control in Power Electronics and Drives, vol. 531 of Studies in Computational Intelligence. Springer International Publishing, 2014, ch. Predictive control in power electronics and drives: Basic concepts, theory, and methods, pp. 181-226.

[16] J. Rodriguez, M. P. Kazmierkowski, J. R. Espinoza, P. Zanchetta, H. Abu-Rub, H. A. Young, and C. A. Rojas, "State of the art of finite control set model predictive control in power electronics," IEEE Trans. Ind. Informat., vol. 9, DOI 10.1109/TII.2012.2221469, no. 2, pp. 10031016, May. 2013.

[17] C. Bordons and C. Montero, "Basic principles of mpc for power converters: Bridging the gap between theory and practice," IEEE Ind. Electron. Mag., vol. 9, DOI 10.1109/MIE.2014.2356600, no. 3, pp. 3143, Sep. 2015.

[18] P. Cortes, J. Rodriguez, D. Quevedo, and C. Silva, "Predictive current control strategy with imposed load current spectrum," IEEE Trans. Power Electron., vol. 23, DOI 10.1109/TPEL.2007.915605, no. 2, pp. 612-618, Mar. 2008.

[19] S. Vazquez, J. I. Leon, L. G. Franquelo, J. M. Carrasco, O. Martinez, J. Rodriguez, P. Cortes, and S. Kouro, "Model predictive control with constant switching frequency using a discrete space vector modulation with virtual state vectors," in 2009 IEEE International Conference on Industrial Technology (ICIT), 2009, DOI 10.1109/ICIT.2009.4939728, Feb. 2009, pp. 1-6.

[20] R. O. Ramirez, J. R. Espinoza, F. Villarroel, E. Maurelia, and M. E. Reyes, "A novel hybrid finite control set model predictive control scheme with reduced switching," IEEE Trans. Ind. Electron., vol. 61, DOI 10.1109/TIE.2014.2308137, no. 11, pp. 5912-5920, Nov. 2014.

[21] R. Aguilera, P. Acuna, P. Lezana, G. Konstantinou, B. Wu, S. Bernet, and V. Agelidis, "Selective harmonic elimination model predictive control for multilevel power converters," IEEE Trans. Power Electron., vol. PP, DOI 10.1109/TPEL.2016.2568211, no. 99, pp. 1-1, 2016.

[22] L. Tarisciotti, P. Zanchetta, A. Watson, S. Bifaretti, and J. C. Clare, "Modulated model predictive control for a seven-level cascaded hbridge back-to-back converter,' IEEE Trans. Ind. Electron., vol. 61, DOI 10.1109/TIE.2014.2300056, no. 10, pp. 5375-5383, Oct. 2014.

[23] M. G. Judewicz, S. A. Gonzalez, N. I. Echeverria, J. R. Fischer, and D. O. Carrica, "Generalized predictive current control (gpcc) for grid-tie three-phase inverters," IEEE Trans. Ind. Electron., vol. PP, DOI 10.1109/TIE.2015.2508934, no. 99, pp. 1-1, 2015.

[24] W. Song, Z. Deng, S. Wang, and X. Feng, "A simple model predictive power control strategy for single-phase pwm converters with modulation function optimization," IEEE Trans. Power Electron., vol. 31, DOI 10.1109/TPEL.2015.2481323, no. 7, pp. 5279-5289, Jul. 2016.

[25] S. Vazquez, A. Marquez, R. Aguilera, D. Quevedo, J. I. Leon, and L. G. Franquelo, "Predictive optimal switching sequence direct power control for grid-connected power converters," IEEE Trans. Ind. Electron., vol. 62, DOI 10.1109/TIE.2014.2351378, no. 4, pp. 2010-2020, Apr. 2015.

[26] J. I. Leon, S. Kouro, L. G. Franquelo, J. Rodriguez, and B. Wu, "The essential role and the continuous evolution of modulation techniques for voltage-source inverters in the past, present, and future power electronics," IEEE Trans. Ind. Electron., vol. 63, DOI 10.1109/TIE.2016.2519321, no. 5, pp. 2688-2701, May. 2016.

[27] J. I. Leon, S. Vazquez, R. Portillo, L. G. Franquelo, J. M. Carrasco, S. Kouro, and J. Rodriguez, "Two-dimensional modulation tech- 
nique for multilevel cascaded h-bridge converters," in 2009 IEEE International Conference on Industrial Technology (ICIT), 2009, DOI 10.1109/ICIT.2009.4939733, Feb. 2009, pp. 1-6.

[28] J. I. Leon, S. Vazquez, R. Portillo, L. G. Franquelo, and E. Dominguez, "Two-dimensional modulation technique with dc voltage control for single-phase two-cell cascaded converters," in 2010 IEEE International Conference on Industrial Technology (ICIT), 2010, DOI 10.1109/ICIT.2010.5472513, Mar. 2010, pp. 1365-1370.

[29] J. I. Leon, S. Kouro, S. Vazquez, R. Portillo, L. G. Franquelo, J. M. Carrasco, and J. Rodriguez, "Multidimensional modulation technique for cascaded multilevel converters," IEEE Trans. Ind. Electron., vol. 58, DOI 10.1109/TIE.2010.2048833, no. 2, pp. 412-420, Feb. 2011.

[30] S. Kouro, M. A. Perez, J. Rodriguez, A. M. Llor, and H. A Young, "Model predictive control: Mpc's role in the evolution of power electronics," IEEE Ind. Electron. Mag., vol. 9, DOI 10.1109/MIE.2015.2478920, no. 4, pp. 8-21, Dec. 2015.

[31] S. A. Larrinaga, M. A. R. Vidal, E. Oyarbide, and J. R. T. Apraiz, "Predictive control strategy for dc/ac converters based on direct power control," IEEE Trans. Ind. Electron., vol. 54, DOI 10.1109/TIE.2007.893162, no. 3, pp. 1261-1271, Jun. 2007.

[32] M. Ciobotaru, R. Teodorescu, and F. Blaabjerg, "A new singlephase pll structure based on second order generalized integrator," in Power Electronics Specialists Conference, 2006. PESC '06. 37th IEEE, DOI 10.1109/PESC.2006.1711988, Jun. 2006, pp. 1-6.

[33] "IEEE recommended practice and requirements for harmonic control in electric power systems," IEEE Std 519-2014 (Revision of IEEE Std 519-1992), DOI 10.1109/IEEESTD.2014.6826459, pp. 1-29, Jun. 2014.

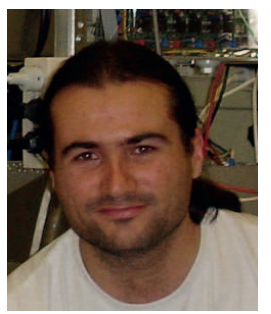

Sergio Vazquez (S'04, M'08, SM'14) was born in Seville, Spain, in 1974. He received the M.S. and $\mathrm{PhD}$ degrees in industrial engineering from the Universidad de Sevilla (US) in 2006, and 2010. He is an Associate Professor at US. His research interests include modeling, modulation and control of power electronics converters for renewable energy systems. Dr. Vazquez was recipient as coauthor of the 2012 Best Paper Award of the IEEE Transactions on Industrial Electronics and 2015 Best Paper Award of the IEEE Industrial Electronics Magazine. He is currently serving as an Associate Editor of the IEEE Transactions on Industrial Electronics.

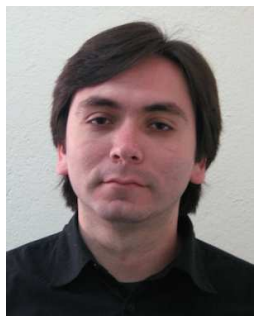

Ricardo P. Aguilera (S'01, M'12) received his M.Sc. degree in Electronics Engineering from the Universidad Tecnica Federico Santa Maria (UTFSM), Chile, 2007, and Ph.D. degree in Electrical Engineering from The University of Newcastle (UN), Australia, 2012. In 2012 he was a Research Academic at UN, where he was part of the Centre for Complex Dynamic Systems and Control. In January 2013, he joined The University of New South Wales (UNSW), Australia, where he currently holds a Senior Research Associate position. His main research interests include power electronics, and theoretical and practical aspects on model predictive control.

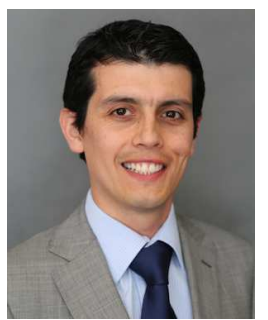

Pablo Acuna (M'12) received the B.S. in Electronics Engineering, the Electronics Engineering Professional, and the Ph.D. degrees in Electrical Engineering from the University of Concepcion, Chile, in 2004, 2007, and 2013 respectively. $\mathrm{He}$ is currently Research Associate at University of New South Wales, Australia. His research interests include electrical power conversion systems and its applications to industry, transportation and utility.

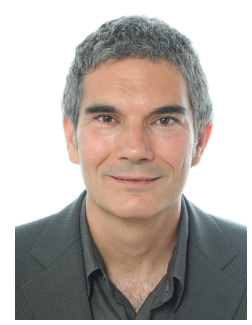

Josep Pou (S'97, M'03, SM'13) received the M.S., and Ph.D. degrees in electrical engineering from the Technical University of Catalonia (UPC), Spain, in 1996, and 2002. In 1990, he joined the faculty of UPC where he became an Associate Professor in 1993. Since February 2013, he is a Professor with the University of New South Wales (UNSW), Australia. He was a Researcher at the Center for Power Electronics Systems, Virginia Tech, Blacksburg in 2001 and 2005. In 2012, he was a Researcher at UNSW. Since 2006, he has collaborated with TECNALIA Research \& Innovation as a research consultant. He has authored more than 200 published technical papers and has been involved in several industrial projects and educational programs in the fields of power electronics and systems. His research interests include modulation and control of power converters, multilevel converters, renewable energy generation, energy storage, power quality, and HVDC transmission systems.

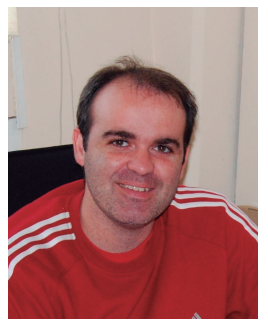

Jose I. Leon (S'04-M'07-SM'14) was born in Cadiz, Spain. He received the M.S. and PhD degrees in telecommunications engineering from Universidad de Sevilla (US), Spain, in 2001 and 2006. He is an Associate Professor at US. His research interests include modulation and control of power converters and renewable energy applications. He was recipient as co-author of the 2008 Best Paper Award of the IEEE Industrial Electronics Magazine, 2012 Best Paper Award of the IEEE Transactions on Industrial Electronics and 2015 Best Paper Award of the IEEE Industrial Electronics Magazine. He was the recipient of the 2014 IEEE Industrial Electronics Society Early Career Award and is currently serving as an Associate Editor for the IEEE Transactions on Industrial Electronics.

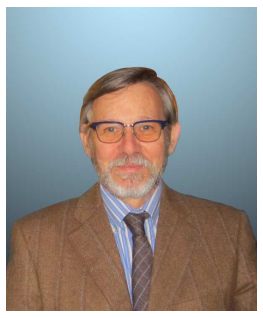

Leopoldo G. Franquelo (M'84-SM'96-F'05) born in Malaga, Spain. He received the M.Sc. and $\mathrm{Ph} . \mathrm{D}$. degrees in electrical engineering from the Universidad de Sevilla, Spain in 1977 and 1980. Dr. Franquelo is an Industrial Electronics Society (IES) Distinguished Lecturer since 2006, an Associate Editor for the IEEE TIE since 2007, Co-EiC since 2014, and EiC since 2015. He was a Member of the IES AdCom (2002-2003), the VP for Conferences (2004-2007), and the President Elect of the IES (2008-2009). He was the President of the IEEE IES (2010-2011) and currently is IES AdCom Life member. His research interest lies on modulation techniques for multilevel inverters and its application to power electronics for renewable energy systems. He has received three best paper awards from IEEE journals. In 2012 and 2015 he received the Eugene Mittelmann and the Antohny J. Hornfeck Service Awards from IES.

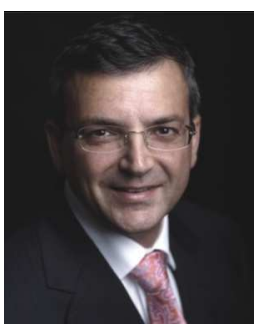

Vassilios G. Agelidis (SM'00, F'16) was born in Serres, Greece. He received the M.S. degree in applied science from Concordia University, Canada, in 1992; and the Ph.D. degree in electrical engineering from Curtin University, Australia, in 1997. He was with Curtin University (1993-1999); the University of Glasgow, U.K. (2000-2004); Murdoch University, Australia (2005-2006); and the University of Sydney, Australia (2007-2010). He is currently the Director of the Australian Energy Research Institute, School of Electrical Engineering and Telecommunications, University of New South Wales (UNSW), Australia. Prof. Agelidis received the Advanced Research Fellowship from the U.K.s Engineering and Physical Sciences Research Council in 2004. He was the Vice President for Operations with the IEEE Power Electronics Society (PELS) from 2006 to 2007 and was an AdCom Member of the IEEE PELS from 2007 to 2009. 